\title{
PRODROMAL SYMPTOMS OF RECURRENT MAJOR DEPRESSIVE EPISODES: A Qualitative Analysis
}

\author{
Bonnie M. Hagerty, Ph.D., R.N., Reg A Williams, Ph.D., R.N., Michelle Liken, M.S., R.N.
}

In four focus groups, 16 people with major depressive or bipolar disorder were asked to describe the onset of an acute depressive episode. Findings indicate that those entering a major depressive episode often have difficulty identifying prodromal symptoms, although they experience early warning phases. These phases and described, and implications for clinical intervention are outlined.

$\mathrm{E}^{\mathrm{n}}$ pisodes of major depression, whether as a component of major depressive disorder or a bipolar disorder, are now recognized as a public-health problem causing untold personal suffering and socioeconomic costs. These episodes are recurrent for most individuals with bipolar disorder and for $50 \%-85 \%$ of those with major depressive disorder (MDD) (Consensus Development Panel, 1985). Clinicians treat more patients for recurrent than first-time episodes (Thase, 1992). Recurrence is associated with increased severity of episodes, increased duration of episodes, and shorter periods of wellness between episodes (Kupfer, 1991; Thase, 1992).

Yet, little is known about the clinical phenomenology of the prodromal phase of a recurrent major depressive episode (MDE). It is unclear what patients begin to experience that later becomes identified as the beginning of the depressive episode, how they come to label this as another episode of depression, and why and when they initiate self-care or seek help. If symptom pat- terns can be identified early as prodromes of reemerging depression, it may be possible to intervene so as to prevent or minimize the episode. Thus, the purpose of this qualitative study was to explore the experience of individuals in the prodromal phase of recurrent MDE.

The prodromal stage of an illness is usually considered a time period during which signs and symptoms of a particular disorder begin to be manifested. These early indications may be quantitatively or qualitatively different than symptoms evident during the acute stage of the illness. They may be fewer, less intense, or different than the symptoms that define the full-blown clinical manifestation of the illness. Prodromal phases of illness vary in duration and may last for only several minutes or hours to many weeks or months.

In general care, assessment and identification of the prodrome of an illness usually allow for timely intervention with the patient that can facilitate prevention of the acute episode, a less protracted course, and

A revised version of a paper submitted to the Journal in May 1995. Authors are at the School of Nursing, University of Michigan, Ann Arbor. 
less invasive and costly treatment. In psychiatry, much of the research on identifying and understanding the prodromal phase has focused on schizophrenia. To date, this research has demonstrated that detection and recognition of the early signs and symptoms of schizophrenia, prior to onset of the acute phase, allows for more timely and effective intervention that can prevent or minimize acute psychosis (Herz, 1985; $O^{\prime}$ Connor, 1991).

There is, however, a paucity of research about the prodromal phase of MDD. Several early studies demonstrated that there was a rapid onset of depressive symptoms when episodes were associated with bipolar disorder and endogenous subtype of depression (Hays, 1964; Hays \& Steinert, 1969). In a more recent study, Young and Grabler (1985) reported that rapid onset of symptoms was associated with endogenous subtype, absence of past or current affective disorders, older age of first depressive episode, less stressful events before the episode, and shorter hospitalization. However, the small number of subjects $(N=11)$ and the retrospective design of the study may limit the generalizability of the findings. Terao (1993) presented a two-case report in which lithium was selfadministered when prodromal symptoms appeared, thus preventing a recurrent episode of depression. This is consistent with Fava and Kellner's (1991) premise that early recognition of prodromal symptoms could be valuable. Kupfer, Frank, and Perel (1989) agreed that early intervention during the prodromal stage of the illness is important in preventing or minimizing relapse and recurrence.

There is evidence to suggest that the duration and symptoms of successive prodromes of depressive disorders are somewhat consistent within individuals (Young Fogg, Scheftner, \& Fawcett, 1990). In addition, it appears that patients generally have at least one psychiatric symptom prior to the onset of depressed mood. Prodromal symptoms of MDE have been reported to include generalized anxiety, sadness, irritability, impaired work, decreased initiative, loss of interest, fatigue, and initial and delayed insomnia (Fava, Grandi, Canestrari, \& Molnar, 1990).

Unfortunately, much of the information about prodromal symptoms of depressive episodes has been gathered retrospectively, from small numbers of patients, utilizing checklists and instruments that list primarily the key symptoms of the acute phase of the disorder. If, indeed, the prodromal phase of MDE might be qualitatively as well as quantitatively different, research should take into account the possibility that symptoms, other than those used as the standard criteria for depression, could portend a depressive episode. Additionally, ways in which patients experience the reemergence of the depression, including temporality of types and severity of symptoms, and the manner in which they interpret the symptoms, label them as depression, and initiate self-care or help-seeking, remain undocumented.

To address this gap, the following research questions were posed in this study: 1) What are the signs and symptoms, and their order and time of occurrence, that patients experience in the prodromal phase of recurrent depression? 2) How do patients with recurrent episodes of major depression respond to prodromal symptoms of recurrence?

\section{METHODS}

This study was qualitative, using focus groups and grounded theory methods (Glaser \& Strauss, 1967). A series of four focus groups took place over the course of one year. The first three groups were conducted via a semistructured interview schedule based on the research questions; responses were then analyzed and hypotheses formulated. In the fourth focus group, the hypotheses were discussed with the members (two of whom had been present at previous focus groups) to assure reliability of findings from the first three groups. Data satu- 
ration was evident in that responses were repeatedly present or notably absent in all descriptions of prodromal experiences provided by participants.

\section{Sample and Procedure}

A total of 16 participants who had been treated for either bipolar disorder or MDD and had had at least two episodes of depression participated in the focus groups. Participants ranged in age from 29 to 65 years. Forty-four percent were women. All participants had been or were in treatment for their disorder. Two were in the midst of an episode of major depression, one was hypomanic, and the remaining participants were at various stages of recovery. All except one had experienced MDEs within the past year. The focus groups were led by two doctoral-prepared psychiatric nurse clinicians and were audiotaped and videotaped. Audiotapes were transcribed and analyzed using ATLAS/Ti (Muhr, 1994), a computer-aided text interpretation and theory-building software program.

\section{RESULTS}

The following recurrent themes were identified: l) "something's not right," 2) "something's really wrong," 3) "the crash," and 4) "getting connected." These themes also could be considered progressive stages within the prodromal phase of a depressive episode.

\section{Something's Not Right}

This theme related to participants' comments about the way in which they experienced initial symptoms of recurrent depression. The duration of this early stage varied, ranging from days to months both between and within individuals across episodes. Within this theme, the following subthemes were identified: $a$ ) denial of symptoms; $b$ ) attributing symptoms to external factors (stress/weather); and c) putting up a front.

All participants described denial of early depressive symptoms. The denial was rein- forced by the fact that depressed or sad mood was usually not the first symptom people experienced. For example, one participant said:

I really didn't want to admit it. I never do want to admit this depression.

Participants described initial negative, uncomfortable feelings that were vague and nondescript, such as fatigue and feeling overwhelmed. These early symptoms tended to be different, both quantitatively and qualitatively, than those used as standard criteria for diagnosing depression. At the time they experienced these symptoms, the subjects attributed them to external factors. Only two participants, at the time of their symptoms, were able to identify these as possible precursors to a depressive episode. As one subject put it:

At first, the symptoms can just be ignored. I can say, "It isn't that serious-like oversleeping or skipping meals or whatever." I can justify it somehow.

Participants also described expending energy to put up a front. They stated that they behaved normally, although they were feeling stressed, exhausted, and unwell. One subject said

Well, there's a certain amount of guilt associated when you feel a little bit phony because you are acting [like] the normal person, your normal state, but you're not feeling the same.

Participants were quick to point out that, in this first stage, symptoms would remit at certain times but not at others. In other words, they did not necessarily experience, in each incident, a recurrent depressive episode subsequent to this first stage.

\section{Something's Really Wrong}

After the initial period of not feeling well, participants described more standard, focused symptoms. The variety of symptoms mentioned during this early phase of recurrence included alterations in sleep and appetite, lack of energy, changes in cognition, withdrawal and isolation, general anxiety, and suicidal ideation. These symptoms were all linked to one general outcome, the inability to experience congru- 
ence, harmony, or synchrony with the environment. This experience, which we labeled as "desynchrony," was disturbing to all participants. They described being on different schedules than those around them, experiencing differences in sleep-wake cycles, having reduced cognitive clarity to match their everyday tasks, and functioning and feeling out of tune with others. These symptoms often led to avoidance of interpersonal interactions. One participant described it as follows:

The first symptom I experienced was I just started waking up early. You know, wake up at two in the morning, wide awake - not to where you're going to go back to sleep again - and you start worrying about the next day, and then, if you're not sleeping, you worry about not sleeping. It's a vicious cycle.

\section{The Crash}

The disturbing experience of desynchrony was followed by the occurrence of one to three specific symptoms that signaled the beginning of the acute episode of depression to all participants. Each of the focus-group members experienced these "pivotal symptoms," which generally encompassed difficulties with sleep, cognition, interpersonal relationships, and safety. Although these symptoms were different for individuals, they generally remained consistent for each person across episodes, although not all occurred with each episode. For example, one participant reported:

My sleep is not the usual. When I am depressed, I oversleep.

\section{Another noted:}

For me, it's the sleeping and the withdrawing [that] are a key that something's wrong.

\section{Yet another stated:}

And my mental acuity also went. I worked as a health care professional and it just scared the bee-gee-bees out of me, the lack of concentration I had at work. I knew I could make a mistake that could really hurt someone.

All participants agreed that once the pivotal symptoms were experienced, the acute episode was inevitable. Pivotal symptoms were the key in alerting them to the onset of MDE. Many described this experience as a rapid spiraling down, from which there was no relief.

\section{Getting Connected}

The fourth theme identified by participants was the need to seek help. Most focus-group members discussed their experiences in this regard with anger, frustration, urgency, and disappointment. All described a critical need at this stage to find a person who would listen to them, someone who could provide feedback about their status and assistance with active intervention. This was usually a family member, a friend, or a therapist who was able to validate their symptoms. The account of one of the men in the group was typical:

I usually think I'm at the end of my rope and I'll talk to my wife. She'll say, "Well, you're no different than you were this other time. You don't remember how bad you were sounding then. You sound the same now; you don't sound any worse." So there's some comfort in that.

Participants also described the importance of finding assistance that they felt was beneficial to them. Focus-group members told of positive experiences that assisted them in reaching out to others, but they spoke as well of experiences that served to maintain, or even exaggerate, their disconnection. When those who had sought help, advice, or validation from family, friends, or health-care providers felt that it was not forthcoming, the resulting disappointment and uncertainty often contributed to the severity and duration of the depressive episode. One participant poignantly recalled an early incident of this sort:

The first time I was ever put on medicine, an antidepressant, the person that prescribed the medicine did a lackadaisical job of monitoring progress. I did not have much improvement in symptoms at the time. You know, you usually wait for the symptoms to improve. The person did not raise the dose at all, no blood level monitoring. It was almost as though the person was giving me medicine to make me think I had something legitimately wrong with me and even shades of that perception from the person that's supposed to be helping me is rather infuriating because you get that enough from the people in your daily life. 


\section{DISCUSSION}

The findings of this qualitative study suggest that people with recurrent depression often experience a specific prodromal phase before the onset of acute depression. This phase varies in duration from episode to episode, ranging from days to weeks, and differs in intensity as well. It appears that the prodromal phase may be resolved without the person ever experiencing an acute episode of depression. This seemed to be the case when the individual was able, early on, to mobilize assistance or self-care, although at times the rationale for absence of a subsequent acute episode was not clear. Some early signs may have been symptom flurries, ordinary responses to stress, or exacerbation of underlying residual symptoms. If, however, a depressed individual reached the point of experiencing pivotal symptoms, a rapid downward spiral signaled the initiation of the acute phase of the illness.

Recent longitudinal studies on the course of depression reveal that $20 \%$ or more of those who experience MDD will not have recovered after one year (Keller et al., 1992). Ongoing residual or dysthymic symptoms signal poor prognoses for persons with MDD (Wells, Burman, Rogers, Hays, \& Camp, 1992). The purpose of the present study was not to compare specific differences between participants who experienced low-level depressive symptoms over time and those who did not, but rather to examine symptomatic change, that is, description of the prodromal phase in which the patient experienced change in severity or type of symptomatology.

Initial prodromal symptoms appear to be qualitatively different than standard depression criteria, resulting in descriptions of vague, diffuse difficulty, but not severe impairment, in one or more spheres of functioning. Fava and Kellner (1991) noted that other kinds of symptoms may confound depressive prodromal research, including premorbid traits, normal subclinical fluctuation of symptoms, and residual symptoms of a previous episode. While such symptoms may interfere with assessment of prodromal occurrence, all focusgroup participants in the present study reported that they perceived their status to be changing at this early stage ("something's not right").

Thus, clinically, the classification of these experiences may be less relevant than the fact that: l) they are perceived by the patient to be uncomfortable and disturbing; 2) they frequently herald the early phase of a recurrent depression; and 3) patients have great difficulty identifying and assessing these symptoms as warnings of depression reemergence, but tend instead to attribute them to external causes such as stress at work or lack of sleep. The exact mechanisms (e.g., psychodynamic, cognitive, neuroregulatory, or neuroendocrine) that interfere with a patient's ability to identify and label this early stage of the prodrome require further investigation. Investigation of potential biological markers of prodromal signs of relapse may provide additional useful information about this stage.

During the second stage of the prodrome ("something's really wrong"), patients begin to associate their symptoms with depression. This stage is characterized by desynchrony and the symptoms involved tend to be the more standard symptoms of MDD, including those affecting mood, sleep, appetite, cognition, social functioning, energy, and suicidal ideation. These symptoms are consistent with those identified by Fava and associates (1990) as indicative of a depressive prodrome. Symptoms that promote the experience of desynchrony seem to be especially important in promoting recognition of the oncoming depression.

While the symptoms contributing to the experience of desynchrony may vary widely from episode to episode, an individual's pivotal signs, usually one to three symptoms, tend to remain consistent across episodes, although all may not appear in the same episode. These pivotal symptoms 
are indicative of "the crash" and signify that the acute phase of the depression has occurred.

It is in these second and third stages that patients actively acknowledge symptoms and may initiate action to address them, either through self-help strategies or helpseeking behavior. Those in the present study who did not immediately attribute their symptoms to depression, and who did not take action, subsequently went on to experience their pivotal symptoms and spiral into the acute phase of the episode. Individuals who had supportive others, including family, friends, or professionals, who were able to point out their symptoms at this stage and encourage helping behavior, tended to report a greater degree of control over their emerging episodes. In spite of this general pattern, some episodes with rapid onsets were perceived by participants as uncontrollable.

Getting connected, having someone listen, and having symptoms validated as depression appear to be important elements of symptom relief. Once individuals "go public" with their symptom difficulties and reach out for assistance, it is important to maintain that connection and focus on the factors that patients view as critical at that point. Often, these may be basic needs such as activities of daily living, assistance organizing and setting priorities, and getting questions answered. All participants described the need to feel as though they had support and options available to fight the depression and achieve relief. They emphasized the importance of mental health professionals listening to them, acknowledging their symptoms, and giving them information about their illness and their medications.

The findings of this study support Karp's (1994) exploration of the ways in which depression as a chronic illness requires an ongoing definition of self. The "something isn't right" phase, identified in the present study, is similar to a phase described by Karp in which patients experienced "in- choate feelings" and the inability to label the experience as depression, attributing their bad feelings to an external situation rather than to themselves. The "something is really wrong" phase is consistent with Karp's description of a "self-working-badly" stage in which patients come to recognize their symptoms as depression. Karp's crisis phase, the point at which patients go public with their disorder and seek help, is analogous to "the crash" and "getting connected" phases identified in this study. While the focus of this research was on describing and understanding the prodromal phase of recurrent depression, Karp sought to explore the issues involved in coming to grips with an illness identity over time. Understanding the need to reconstruct and reinterpret one's past in terms of the current situation-constructing a theory of the nature of depression as life-long and establishing modes of coping - can help to clarify the work involved in identifying and labeling symptoms of recurrent depression.

While subjects were asked to recall information retrospectively, the consistency of responses from all participants, including those with bipolar diagnoses, was striking. The findings of this study are a step toward identifying specific characteristics of the prodromal phase of recurrent depression and patient responses, and toward a more comprehensive phenomenological description of the experience and characteristics of this phase, which can inform further investigations of the course of recurrent depression.

The present findings also suggest that specific clinical interventions can be developed to identify early warning signs and initiate actions that can help minimize or prevent a major depressive episode. Such interventions may include psychoeducation about recurrent depression and the individual's specific prodromal patterns and pivotal symptoms; monitoring techniques using standardized tools; involvement of significant others in monitoring and responding to early depressive symptoms; 
and specific strategies designed to assist patients to feel connected and hopeful about their plight. Lastly, the importance that participants attributed to mental health professionals listening to their symptoms, showing concern, and providing encouragement cannot be overemphasized.

\section{REFERENCES}

Consensus Development Panel. (1985). Mood disorders: Pharmacologic prevention of recurrences. American Journal of Psychiatry, 142, 469-475.

Fava, G.A., Grandi, S., Canestrari, R., \& Molnar, G. (1990). Prodromal symptoms in primary major depressive disorder. Journal of Affective Disorders, 19, 149-152.

Fava, G.A., \& Kellner, R. (1991). Prodromal symptoms in affective disorders. American Journal of Psychiarry, 148, 823-830.

Glaser, B.G., \& Strauss, A.L. (1967). The discovery of grounded theory: Strategies for qualitative analysis. New York: Aldine deGruyter.

Hays, P. (1964). Modes of onset of psychotic depression. British Medical Journal, 2, 779-784.

Hays, P., \& Steinert, J. (1969). A blind comparative trial of nortriptyline and isocarboxazid in depressed outpatients. Canadian Psychiatric Association Journal, 14, 307-311.

Herz, M. (1985). Prodromal symptoms and prevention of relapse in schizophrenia, Journal of Clinical Psychiatry, 46(11), 22-25.

Karp, D.A. (1994). Living with depression: Illness and identity turning points. Qualitative Health Research, 4(1), 6-30.

Keller, M.B., Lavori, P.W., Mueller, T.I., Endicott, J., Coryell, W., Hirschfeld, R.M.A., \& Shea, T. (1992). Time to recovery, chronicity, and levels of psychopathology in major depression. Archives of General Psychiatry, 49, 809-816.

Kupfer, D.J. (1991). Long-term treatment of depression. Journal of Clinical Psychiatry, 52(5), 28-34.

Kupfer, D.J., Frank, E., \& Perel, J.M. (1989). The advantage of early treatment interaction in recurrent depression. Archives of General Psychiatry, 46, $771-775$.

Muhr, T. (1994). ATLAS/Ti: Computer-aided text interpersonal and theory buildings. Berlin, Germany: Author.

O'Connor, F. (1991). Symptom monitoring for relapse prevention in schizophrenia. Archives of Psychiatric Nursing, 5(4), 193-201.

Terao, T. (1993). Prodromal symptoms of depression and self-administration of lithium. Biological $P_{s y-}$ chiatry, 34(3), 198-199.

Thase, M.E. (1992). Long-term treatment of recurrent depressive disorders. Journal of Clinical Psychiatry, 53(9), 32-44.

Wells, K.B., Burnam, A., Rogers, W., Hays, R., \& Camp, P. (1992). The course of depression in adult outpatients. Archives of General Psychiatry, 49, 788-794.

Young, M.A., Fogg, L.F., Scheftner, W.A., \& Fawcett, J.A. (1990). Concordance of symptoms in recurrent depressive episodes. Journal of Affective Disorders, 20, 79-85.

Young, M.A., \& Grabler, P. (1985). Rapidity of symptom onset in depression. Psychiatry Research, 16, 309-315. 